\title{
Ultrafiltration modérée de lait cru entier froid sur membranes minérales : essais semi-industriels
}

\author{
par \\ M. BENNASAR et B. TARODO DE LA FUENTE
}

\section{Résum é}

Sur la base des connaissances acquises en laboratoire, nous avons réalisé un module pilote d'ultrafiltration d'une surface d'échange de $17,8 \mathrm{~m}^{2}$, qui a été installé dans une entreprise laitière et utilisé pour des concentrations ménagées. Les essais effectués permettent de confirmer et de compléter nos précédents travaux. Nous retrouvons l'influence favorable de la vitesse de circulation du lait sur le débit d'ultrafiltrat et de la température d'emprésurage qui accélère les réactions primaire et secondaire de coagulation et provoque un accroissement supplémentaire de la perméation. La stabilité chimique et bactériologique du rétentat et les performances de la membrane sont vérifiées pour des durées d'opération et des facteurs de concentration proches des conditions industrielles. Les taux de rétentions et les facteurs de concentration des principaux constituants du rétentat sont déterminés. Une relation permettant de calculer un facteur de concentration théorique pour chaque élément en fonction de son taux de rétention moyen est établie et permet d'évaluer la participation des matières azotées et du calcium au colmatage des membranes. L'intérêt économique et pratique de ce nouveau matériau membranaire minéral pouvant être nettoyé et désinfecté sans dommage et avec la même rigueur que les autres installations laitières est démontré.

Mots clés :

Ultrafiltration - Lait.

Titre abrégé :

Ultrafiltration de lait froid sur membranes minérales.

Ce travail a été réalisé dans le cadre d'un contrat A.N.V.A.R., nº A 791100401001. Centre de Génie et Technologie Alimentaire, Laboratoire de Technologie Alimentaire, Université des Sciences et Techniques du Languedoc, place E.-Bataillon 34060 Montpellier. 


\section{S u $\mathrm{m}$ mary}

MODERATE ULTRAFILTRATION OF RAW COLD MILK ON MINERAL MEMBRANES: INDUSTRIAL EXPERIMENTS

An ultrafiltration pilot system with an exchange area of $17,8 \mathrm{~m}^{2}$ has been realized and installed in a dairy manufacture. The experiments had confirmed and completed our previous works. We find the favourable influence of flow rate and renneting temperature on ultrafiltrate flux. The latter speed up primary and secondary clotting reactions and bring about a further increase of permeation. Chemical and bacteriological concentrate stability and membrane performances are confirmed for length of time and concentration factors close to industrial conditions. Retention rates and concentration factors of concentrate main constituents are determined. For every constituent a relationship is drawn in order to calculate theoritical concentration factor from its mean retention rate. This relationship is able to assess the proteins and calcium part in fouling. The economical an practical interest of these new mineral membranes is bring out.

Key words:

Ultrafiltration - Milk.

\section{INTRODUCTION}

Nos précédents travaux exposés lors de la journée "Membranes artificielles et matériaux biologiques » de Montpellier, Bennasar (1981), ont montré que les membranes minérales (céramique) fabriquées par la Société Eurocéral-Vendargues, appartenant à la nouvelle génération des membranes solides, pouvaient être utilisées pour l'ultrafiltration du lait. De récentes communications (Roger et Maubois, 1981 et Caminade 1982) font le point sur les utilisations de ces nouveaux matériaux qui présentent de gros avantages par rapport aux membranes polymères des générations précédentes, avec en particulier une meilleure résistance chimique, mécanique et thermique facilitant leur nettoyage et augmentant leur durée de vie.

Nous avons déterminé, Bennasar (1981) et Bennasar et al. (1982), sur lait cru entier à froid, les conditions optimales de leur utilisation (diamètre moyen des pores, pression de travail, régime d'écoulement du produit, etc.) et les caractéristiques physico-chimiques des rétentats et ultrafiltrats correspondants.

Nous avons ainsi montré que le débit d'ultrafiltrat et le $\mathrm{pH}$ varient peu pour une ultrafiltration modérée en « batch " (facteur de concentration 1,2 fois) limitée à 2 ou $3 \mathrm{~h}$. 
Nous avons aussi mis en évidence que le diamètre moyen des pores des membranes et la pression ont peu d'influence sur ce débit, mais qu'il est par contre directement proportionnel à la vitesse de circulation du lait.

En règle générale, le taux de rétention en matières azotées très élevé $(95 \%)$ ne correspond pratiquement qu'au seul passage du NPN (azote non protéique) et reste constant pendant toute la durée de l'opération. Malgré cela, les performances demeurent insuffisantes étant donné les basses températures mises en œuvre et nous avons recherché d'autres solutions pour les améliorer. C'est ainsi que nous avons mis en évidence que l'emprésurage préalable du lait à basse température $\left(4^{\circ} \mathrm{C}\right)$, et son enrichissement en calcium permettaient d'augmenter notablement le débit d'ultrafiltrat tout en conservant le pouvoir séparatif de la membrane.

Afin d'interpréter ces diverses observations mettant en cause essentiellement la couche de polarisation et le colmatage déjà signalés par de nombreux auteurs : Blatt et al. (1970), Porter et Michaels (1971), Porter (1972), Glover et Brooker (1974), Dejmek (1975), Raymond et Musso (1977), Clifton (1980), Hiddinkk et al. (1980) et N'guyen (1980), nous avons échafaudé une théorie sur leur formation basée sur la charge et la taille des micelles qui en sont quantitativement le constituant majeur.

Sur la base des connaissances ainsi acquises, nous avons conçu et réalisé un module pilote industriel équipé de ces membranes et présentant une surface d'échange de $17,8 \mathrm{~m}^{2}$. Cet appareil pouvant traiter plusieurs milliers de litres de lait a été installé chez un industriel laitier et le présent travail a pour but de vérifier nos précédents résultats brièvement rappelés ci-dessus, de les compléter et de montrer ainsi que le procédé est applicable au stade industriel.

\section{MATERIELS ET METHODES}

\section{Lait et présure}

Le lait de grand mélange est collecté quotidiennement par l'usine. Il est cru, entier et stocké à $3^{\circ} \mathrm{C}$ avant traitement.

La solution de présure est un extrait du commerce de force 10000 (520 mg/1 de chymosine active) et le taux d'emprésurage (25 ml de cette solution pour 100 litres de lait) est celui utilisé couramment pour les fabrications. La solution d'enrichissement en calcium est une solution du commerce (présurol) à $520 \mathrm{~g} / 1$ de chlorure de calcium. 


\section{Méthodes d'analyses}

L'extrait sec total (EST) est déterminé à l'étuve à $103 \pm 1^{\circ} \mathrm{C}$. L'azote total est dosé selon la méthode officielle de Kjeldahl avec un appareil Tecator Kjeltec I et les matières azotées (MAT) correspondantes sont calculées à l'aide du facteur 6,39 .

Les matières grasses (MG) sont déterminées par la méthode photométrique à l'aide d'un appareil Foss-Electric type MTU.

Les chlorures $(\mathrm{Cl})$ sont évalués par méthode potentiométrique avec le chloruromètre Corning.

Le calcium $(\mathrm{Ca})$ est dosé par la méthode complexométrique avec le calcimètre Corning.

Le $\mathrm{pH}$ est mesuré à l'aide d'un $\mathrm{pH}$ mètre Tacussel Minisis.

Le point cryoscopique $(\Delta \mathrm{T})$ est évalué au cryoscope Fiske.

L'indice de lipolyse est déterminé par la méthode BLM (Bovine Lipolys's Method) suivant Mouillet et al. (1981).

Les germes totaux (GT) sont dénombrés sur milieu PCA à $30^{\circ} \mathrm{C}$ et les coliformes (Cf) sur désoxycholate à $37^{\circ} \mathrm{C}$.

\section{Installation d'ultrafiltration (fig. 1)}

Le bac d'alimentation peut contenir jusqu'à 4000 litres de lait maintenus à basse température par agitation et réfrigération de la double enveloppe par un circuit d'eau glacée. L'alimentation du lait est effectuée par une pompe centrifuge $\left(5 \mathrm{~m}^{3} / \mathrm{h}\right.$ à 5 bar); il est ensuite repris avec le lait sortant du module d'ultrafiltration par une pompe centrifuge de recirculation à gros débit $\left(50 \mathrm{~m}^{3} / \mathrm{h}\right.$ à 1 bar) qui permet d'augmenter sa vitesse de passage.

Le module d'ultrafiltration est composé de 2 ou 4 sous-unités alimentées séparément en lait et constituées chacune par l'assemblage en série de tubes unitaires d'ultrafiltration de diamètre moyen des pores 4600 A décrits dans nos précédents travaux : Bennasar (1981).

Quelle que soit la configuration adoptée, la surface membranaire reste constante et égale à $17,8 \mathrm{~m}^{2}$, mais le doublement de la section de passage et la réduction de moitié de la longueur de chaque unité permettent d'augmenter la vitesse de circulation en conservant des pertes de charges semblables. Les manomètres d'entrée et de sortie permettent de connaître cette perte de charge et de régler la différence de pression de part et d'autre de la membrane grâce à la vanne de contre pression.

Le rétentat peut être renvoyé dans le tank d'alimentation ou dans le système d'ultrafiltration en jouant sur les vannes de contre pression et de recirculation.

Le nettoyage et la désinfection sont assurés « en place » grâce à 2 bacs (acide et soude) incorporés dans le circuit. 


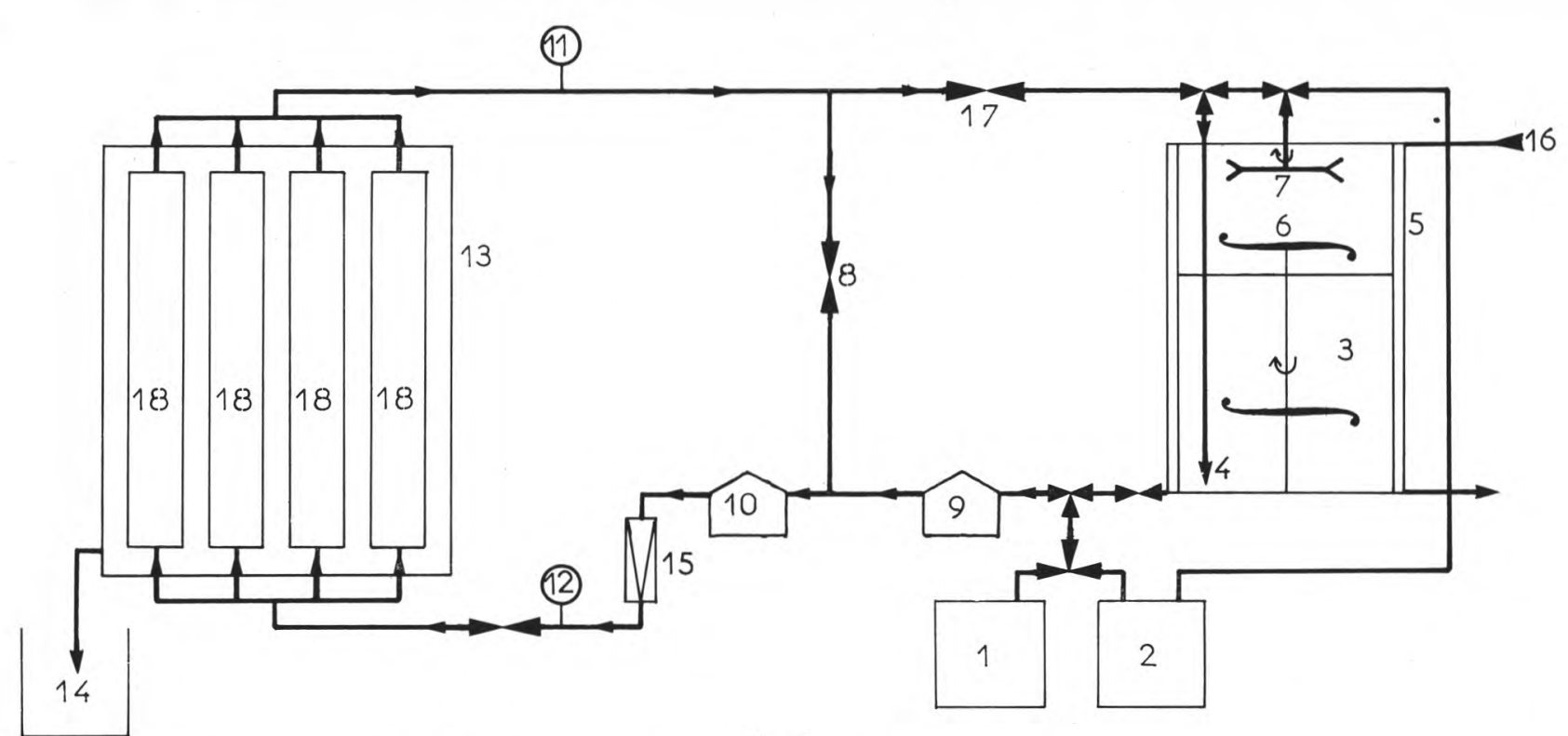

\section{6}

5: Tank alimentation.

6: Agitation.

7: Tourniquet.

8: Vanne recirculation.

9: Pompe centrifuge haute pression (alimentation).

10 : Pompe centrifuge basse pression (recirculation).

11: Manomètre (sortie).

12: Manomètre (entrée).

13: Module d'ultrafiltration.

14: Ultrafiltrat.

15: Débitmètre.

16: Eau glacée.

17: Vanne contre-pression.

18 : Sous-unité d'ultrafiltration.

fig. 1

Installation pilote d'ultrafiltration - Ultrafiltration pilot plant

\begin{tabular}{r|r}
$1:$ Alkaline detergent. & zo \\
$2:$ Acid detergent. & N \\
$3:$ Milk. & o. \\
$4:$ Retentate. & \\
$5:$ Feed tank. & \\
$6:$ Agitation. & \\
$7:$ Turnstile. & \\
$8:$ Recirculation valve. & \\
$9:$ High pressure centrifugal feed pump. \\
$10:$ Low pressure centrifugal recirculation pump. \\
$11:$ Manometer (outlet). \\
$12:$ Manometer (inlet). \\
$13:$ Ultrafiltration modulus. \\
$14:$ Ultrafiltrate. \\
$15:$ Rotameter. \\
$16:$ Iced water. \\
$17:$ Pressure control valve. \\
$18:$ Ultrafiltration unity.
\end{tabular}




\section{RESULTATS}

\section{Etude des paramètres d'ultrafiltration}

Compte tenu de la mise en circulation du lait par une pompe centrifuge, il est impossible de faire varier le régime d'écoulement sans modifier les pressions d'entrée et de sortie et donc d'analyser séparément chacun de ces facteurs.

Nous avons tout de même réussi à étudier l'influence de la vitesse de circulation qui, comme nos travaux précédents l'ont montré : Bennasar et al. (1982), est le principal facteur agissant sur le débit d'ultrafiltrat, en augmentant le nombre de sous-unités d'ultrafiltration mises en service, comme indiqué dans le chapitre matériels et méthodes.

Nous avons ainsi pu faire varier la vitesse de 1 à $3,5 \mathrm{~m} / \mathrm{s}$ dans les conditions suivantes :

Pression entrée : 6 bar

Pression sortie : 1 bar

pertes de charges : 5 bar.

Température : $5 \pm 1^{\circ} \mathrm{C}$.

Le débit d'ultrafiltrat et le taux de rétention des matières azotées sont représentés en fonction de la vitesse sur la figure 2. Nous constatons que le débit augmente régulièrement de 3 à $15 \mathrm{l} / \mathrm{h} \cdot \mathrm{m}^{2}$ quand la vitesse passe de 1 à $3,5 \mathrm{~m} / \mathrm{s}$ alors que le taux de rétention reste stable à sa valeur élevée habituelle ( 94 à $95 \%$ ) dans le même temps.

\section{Etude de l'ultrafiltration de lait cru entier}

Les résultats rapportés dans ce paragraphe représentent la moyenne d'une dizaine d'essais effectués dans les conditions suivantes :

Pertes de charges : 5 bar, vitesse : $3,4 \mathrm{~m} / \mathrm{s}$.

La température fixée initialement à $5^{\circ} \mathrm{C} \pm 1$ a parfois été difficile à maintenir par suite de l'insuffisance du circuit d'eau glacée et a pu atteindre $12^{\circ} \mathrm{C}$ en fin de traitement. Dans tous les cas, le débit d'ultrafiltrat a été corrigé et ramené à la température de référence $\left(5^{\circ} \mathrm{C}\right)$ en tenant compte d'essais précédents montrant qu'il varie d'environ $2 \%$ par ${ }^{\circ} \mathrm{C}$. Nous constatons sur la figure 3 qu'il diminue régulièrement avec la durée de l'opération et l'augmentation de la concentration. De $14,5 \mathrm{l} / \mathrm{h} \cdot \mathrm{m}^{2}$ au départ, il passe à $12,2 \mathrm{l} / \mathrm{h} \cdot \mathrm{m}^{2}$ après environ $5 \mathrm{~h}$ d'ultrafiltration pour un facteur de concentration 1,4 fois, soit une chute moyenne de 3 à $4 \%$ par heure plus accentuée en début d'opération. L'analyse des constituants de l'ultrafiltrat permet de déterminer l'évolution de leurs taux de rétention qui sont indiqués sur la figure 4 en fonction de la concentration du lait. 
Les matières grasses dosées par la méthode photométrique sont absentes à tout instant de l'ultrafiltrat, ce qui correspond donc à un taux de rétention apparent maximum de $100 \%$. Le taux de rétention des matières azotées malgré une légère augmentation passagère au début reste entre 94 et $95 \%$ durant toute l'opération. Celui du calcium diminue légèrement de 73,5 à $72,5 \%$ environ en fin de traitement ainsi que celui de l'extrait sec total qui passe de 59 à $57,5 \%$.

Enfin, le taux de rétention des chlorures est négatif et chute de façon importante pendant toute l'opération $(-7$ à $-11 \%)$ mais surtout au début.

L'analyse de ces mêmes constituants dans le rétentat permet de mesurer leurs facteurs de concentration respectifs qui sont représentés en fonction de la concentration volumique du lait sur la figure 5. Le facteur de concentration des matières azotées qui est le plus élevé atteint 1,37 pour un facteur final de concentration volumique de 1,4 . Les facteurs de concentration en matières grasses, calcium et extrait sec total augmentent aussi linéairement et se situent en fin d'opération respectivement à : $1,30,1,29$ et 1,24 . Celui des chlorures qui est inférieur à 1 chute rapidement au début puis régulièrement jusqu'à 0,95 en fin de traitement.

L'abaissement cryoscopique a tendance à augmenter aussi bien dans le rétentat $(-0,542$ à $-0,566)$ que dans l'ultrafiltrat $(-0,477$ à $-0,494)$ quand le facteur de concentration s'élève comme le montre la figure 6 . En revanche, le $\mathrm{pH}$ varie très peu dans le rétentat $(6,64$ à 6,62) comme dans l'ultrafiltrat $(6,69$ à 6,67$)$ pendant toute la durée de l'opération.

Les contrôles bactériologiques (germes totaux et coliformes) dans le lait initial, le rétentat et l'ultrafiltrat consignés dans le tableau 1 montrent que ces flores ont tendance à se développer dans le rétentat surtout lorsque la température s'élève; par contre lorsque la température basse initiale a pu être correctement maintenue leur prolifération est quasi inexistante puisque leur augmentation correspond pratiquement au facteur de concentration; dans l'ultrafiltrat elles sont en général absentes.

\section{Etude de l'ultrafiltration de lait cru entier préemprésuré}

Le préemprésurage et l'enrichissement en calcium du lait sont effectués avec une solution de présure de force 10000 et de $\mathrm{Ca} \mathrm{Cl}_{2}$ à $520 \mathrm{~g} / 1$ dans les conditions habituelles des fabrications fromagères mentionnées dans le tableau 2.

Les paramètres d'ultrafiltration sont les mêmes que pour le lait cru entier normal et en particulier la température $\left(5^{\circ} \mathrm{C} \pm 1\right)$.

Les résultats obtenus par comparaison avec un lait cru entier témoin (non emprésuré, non additionné de calcium) sont donnés dans le tableau 2. 
TABLEAU 1 - TABLE 1

Evolution de la flore dans différentes conditions d'ultrafiltration

Flora evolution under various ultrafiltration conditions

\begin{tabular}{|c|c|c|c|c|c|c|c|c|c|c|c|c|c|}
\hline \multirow{2}{*}{\multicolumn{2}{|c|}{$\begin{array}{l}\mathrm{t} \\
\mathrm{h}\end{array}$}} & \multicolumn{4}{|c|}{ Lait départ } & \multicolumn{4}{|c|}{ Rétentat } & \multicolumn{4}{|c|}{ Ultrafiltrat } \\
\hline & & $\begin{array}{c}\theta \\
{ }^{\circ} \mathrm{C}\end{array}$ & $\mathrm{pH}$ & $\begin{array}{l}\mathrm{GT} \\
/ \mathrm{ml}\end{array}$ & $\begin{array}{l}\text { Cf } \\
/ \mathrm{ml}\end{array}$ & $\begin{array}{c}\theta \\
{ }^{\circ} \mathrm{C}\end{array}$ & $\mathrm{pH}$ & $\begin{array}{l}\mathrm{GT} \\
/ \mathrm{ml}\end{array}$ & $\begin{array}{l}\mathrm{Cf} \\
/ \mathrm{ml}\end{array}$ & $\begin{array}{c}\theta \\
{ }^{\circ} \mathrm{C}\end{array}$ & $\mathrm{pH}$ & $\begin{array}{l}\mathrm{GT} \\
/ \mathrm{ml}\end{array}$ & $\begin{array}{l}\mathrm{Cf} \\
/ \mathrm{ml}\end{array}$ \\
\hline & 1,4 & 6,4 & 6,68 & $1,5.10^{6}$ & $4,3.10^{3}$ & 10,7 & 6,70 & $1,6.10^{6}$ & $12.10^{4}$ & 10,7 & 6,80 & 10 & 0 \\
\hline & 2,2 & 6,4 & 6,68 & $1,5.10^{6}$ & $4,3.10^{3}$ & 12,8 & 6,70 & $4,2.10^{6}$ & $12,2.10^{4}$ & 12,8 & 6,77 & 10 & 0 \\
\hline & 4,4 & 5,8 & 6,62 & $1,6.10^{6}$ & $12.10^{3}$ & 13,2 & 6,56 & $6.10^{6}$ & $20.10^{4}$ & 13,2 & 6,65 & 0 & 0 \\
\hline & 4,8 & 6,5 & 6,66 & $4,8.10^{6}$ & $40.10^{3}$ & 6,7 & 6,65 & $6,8.10^{6}$ & $46.10^{3}$ & 6,7 & 6,70 & 0 & 0 \\
\hline $\begin{array}{ll}\mathrm{t} & : \\
\theta & : \\
\mathrm{GT} & : \\
\mathrm{Cf} & :\end{array}$ & $\begin{array}{l}\text { dur } \\
\text { tem } \\
\text { ger } \\
\text { coli }\end{array}$ & $\begin{array}{l}\text { d'ultra } \\
\text { ature. } \\
\text { totau } \\
\text { mes. }\end{array}$ & ration. & & & & $\begin{array}{l}\mathrm{t} \\
\theta \\
\mathrm{GT} \\
\mathrm{Cf}\end{array}$ & $\begin{array}{l}\text { rocess ti } \\
\text { emperatn } \\
\text { otal gern } \\
\text { oliforms }\end{array}$ & $\begin{array}{l}\text { ze of ult } \\
\text { e. } \\
\text { s. }\end{array}$ & tratior & & & \\
\hline
\end{tabular}


TABLEAU 2 - TABLE 2

Caractéristiques d'ultrafiltration de lait cru entier préemprésuré

Ultrafiltration features of renneted whole raw milk

\begin{tabular}{|c|c|c|c|c|c|c|c|c|c|}
\hline \multicolumn{3}{|c|}{ Préemprésurage } & Addition & $\mathrm{D}$ & \multicolumn{5}{|c|}{$\mathrm{T} \%$} \\
\hline Taux & $\theta$ & $\mathrm{E}$ & $\mathrm{Ca} \mathrm{Cl}{ }_{2}$ & $1 / \mathrm{hm}^{2}$ & EST & MAT & MG & $\mathrm{Cl}$ & $\mathrm{Ca}$ \\
\hline $\mathrm{ml} / 100 \mathrm{l}$ & ${ }^{\circ} \mathrm{C}$ & $\mathrm{h}$ & $\mathrm{g} / 1$ & & & & & & \\
\hline- & - & - & - & 14,5 & 58,2 & 94,2 & 100 & $-9,8$ & 73,1 \\
\hline 25 & 2,7 & 14,8 & 0,1 & 3,6 & 60,0 & 95,3 & 100 & $-6,8$ & 72,1 \\
\hline 25 & 7,8 & 14,0 & 0,1 & 19,6 & 56,9 & 94,1 & 100 & $-9,9$ & 72,4 \\
\hline \multicolumn{5}{|c|}{$\theta$ : température. } & \multicolumn{5}{|c|}{$\theta:$ temperature. } \\
\hline \multicolumn{5}{|c|}{ E : durée. } & \multicolumn{5}{|c|}{$\mathrm{E}$ : process time. } \\
\hline \multicolumn{5}{|c|}{ D : débit d'ultrafiltrat. } & \multicolumn{5}{|c|}{ D : ultrafiltrate flux. } \\
\hline \multicolumn{5}{|c|}{$\mathrm{T}$ : taux de rétention. } & \multicolumn{5}{|c|}{$\mathrm{T}$ : retention coefficient. } \\
\hline
\end{tabular}


Nous constatons que le débit d'ultrafiltrat augmente de façon très importante avec la température de préemprésurage puisqu'il passe de $3,61 / h^{\prime} \cdot m^{2}$ à $2,7^{\circ} \mathrm{C}$, à $19,61 / \mathrm{h} \cdot \mathrm{m}^{2}$ à $7,8^{\circ} \mathrm{C}$. Cette dernière valeur représente par rapport au témoin $\left(14,5 \mathrm{l} / \mathrm{h} . \mathrm{m}^{2}\right)$ un accroissement de $35 \%$ environ du débit.

Les taux de rétention des divers constituants restent voisins des valeurs habituelles, mais ils ont tendance à varier de façon inverse avec le débit. En ce qui concerne le calcium et les chlorures, l'addition de ce sel modifie les concentrations initiales et rend difficile la comparaison avec le témoin.

\section{Etude du nettoyage et de la désinfection}

Après chaque passage de lait, l'installation d'ultrafiltration est soumise " en place » à un cycle de nettoyage et désinfection comparable à celui mis au point dans notre laboratoire et défini dans nos précédents travaux : Bennasar (1981), mais mettant en jeu les détergents et les températures habituels de l'industrie laitière. C'est ainsi que le détergent alcalin est remplacé par une solution de soude à $3 \%$ et $70^{\circ} \mathrm{C}$, le détergent acide par une solution d'acide nitrique à $3 \%$ à $60^{\circ} \mathrm{C}$ et le désinfectant par une solution d'extrait de javel ( $48^{\circ}$ chlorométrique) à 1 ou $2 \%$ à température ambiante. Ces traitements ont été parfaitement supportés par les membranes et les contrôles de débits pratiqués ensuite sur eau et lait ont donné toutes satisfactions.

\section{DISCUSSION}

La variation du débit d'ultrafiltration en fonction du régime d'écoulement du lait (fig. 2) est tout à fait semblable à celle déjà observée en laboratoire et rapportée dans nos travaux antérieurs : Bennasar et al. (1982).

Nous retrouvons, en particulier, pour des vitesses égales des débits voisins alors que les nombres de Reynolds sont très différents. Il semble donc que la vitesse soit le principal facteur d'amélioration du débit, comme l'a indiqué Gernedel (1980) en montrant l'influence du taux de cisaillement sur l'épaisseur de la couche de dépôt.

La possibilité d'atteindre des vitesses plus élevées qu'en laboratoire $(3,5 \mathrm{~m} / \mathrm{s}$ au lieu de $2,8 \mathrm{~m} / \mathrm{s})$, nous a permis de confirmer l'évolution linéaire du débit au-delà de cette limite et de travailler à des valeurs bien supérieures $\left(15\right.$ 1/h.m ${ }^{2}$ au lieu de 11 1/h.m²).

L'absence de variations du taux de rétention en matières azotées et sa valeur représentées sur cette même figure 2 en fonction du régime, concordent également avec les résultats obtenus en laboratoire et les valeurs habituelles données dans la littérature pour des conditions voisines (Maubois et Mocquot, 1971). 


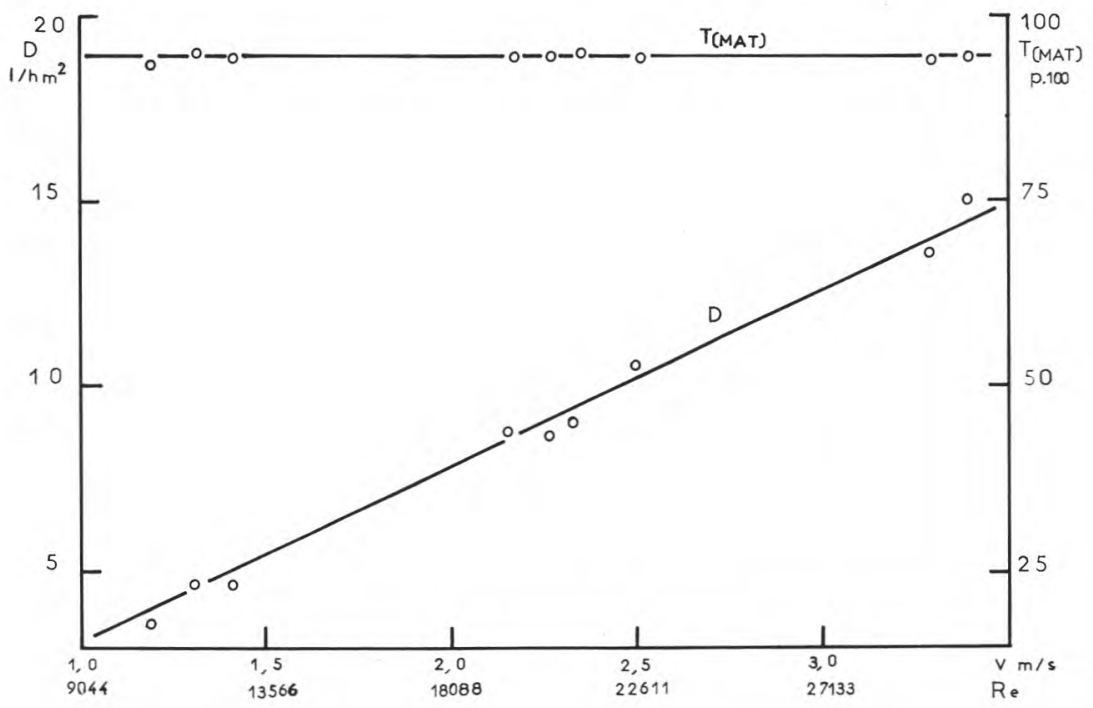

fig. 2

Débit d'ultrafiltrat et taux de rétention en matières azotées en fonction du régime d'écoulement du lait

Influence of milk flow on ultrafiltrate flux and retention coefficient of nitrogenous compounds

D : débit d'ultrafiltrat.

$\mathrm{T}$ : taux de rétention.

$\mathrm{v}$ : vitesse de circulation.

Re : nombre de Reynolds.
D : ultrafiltrate flux.

$\mathrm{T}$ : retention coefficient.

$\mathrm{v}$ : circulation rate.

Re : Reynolds number.

La chute du débit d'ultrafiltrat dans le temps et avec l'accroissement de la concentration (fig. 3) vérifie pour des durées supérieures de traitement ( $5 \mathrm{~h}$ au lieu de $2 \mathrm{~h}$ ) nos précédents résultats, mettant en évidence un colmatage progressif et inévitable de la membrane observé également sur lait entier par Yan et al. (1979).

Nous avons établi qu'il existait entre le taux de rétention et le facteur de concentration d'un même élément dans le rétentat la relation théorique suivante :

$$
\mathrm{F}_{\mathrm{t}(\mathrm{x})}^{\prime}=\underset{\mathrm{m}(\mathrm{x})}{\mathrm{T}}(\mathrm{F}-1)+1
$$

où $\mathrm{F}_{\mathrm{t}(\mathrm{x})}^{\prime}=$ facteur de concentration théorique de l'élément $\mathrm{x}$

Le facteur de concentration réel de l'élément $\mathrm{x}\left(\mathrm{F}_{\mathrm{x}}^{\prime}\right)_{\mathrm{x}}$ étant quant
à lui mesuré par le rapport $\frac{\mathrm{C}_{\mathrm{r}}(\mathrm{x})}{\mathrm{C}_{1}(\mathrm{x})}$ avec : 


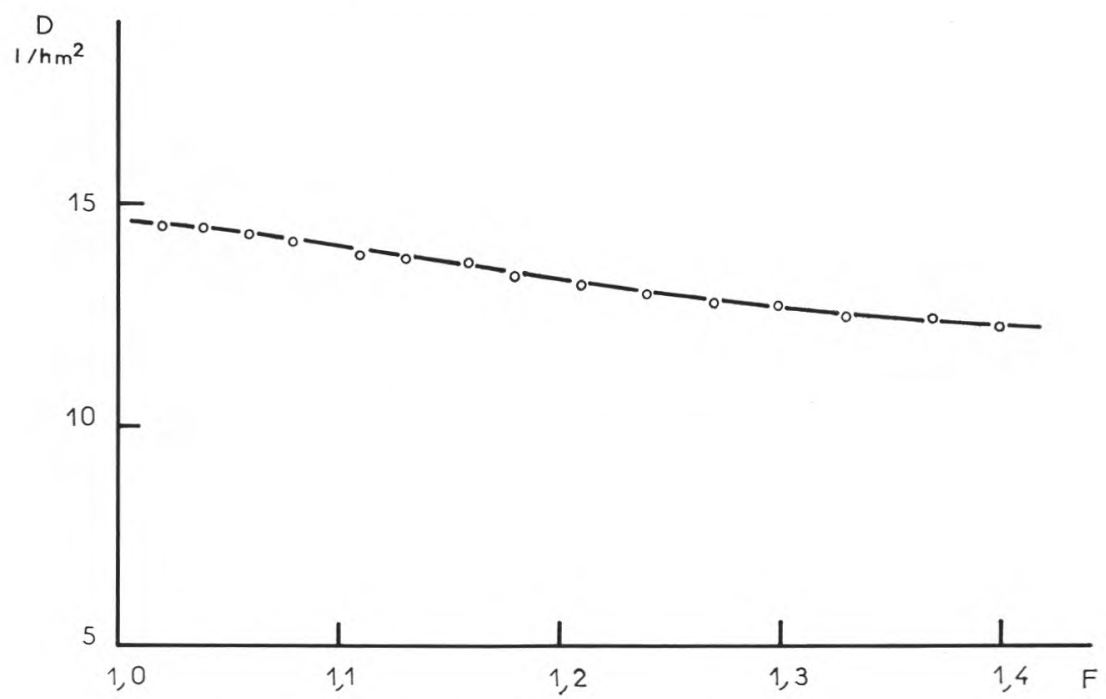

fig. 3

Débit d'ultrafiltrat en fonction du facteur de concentration

Ultrafiltrate flux as a function of concentration factor

D : débit d'ultrafiltrat.

F : facteur de concentration volumique.
D : ultrafiltrate flux.

$\mathrm{F}$ : volumetric concentration factor.

\section{TABLEAU $3-T A B L E 3$}

Facteurs de concentration expérimentaux et théoriques en fonction des taux de rétention moyens des différents éléments du rétentat

(concentration volumique finale réelle $F=1,41$ )

Tm : taux de rétention moyen.

$\mathrm{F}^{\prime}$ : facteur de concentration expérimental.

$F^{\prime} t$ : facteur de concentration théorique.

Experimental and theoretical concentration factors evolution as a function of mean retention coefficients of different retentate elements (real final volumetric concentration $F=1,41$ )

Tm : mean retention coefficient.

$F^{\prime}$ : experimental concentration factor.

F't : theoretical concentration factor.

\begin{tabular}{l|c|c|c}
\hline \multicolumn{1}{r|}{ Eléments } & Tm & F't & F' \\
\cline { 2 - 3 } MAT & 94,2 & 1,39 & 1,37 \\
Ca & 73,1 & 1,30 & 1,29 \\
MG & 100 & 1,41 & 1,30 \\
Cl & $-9,75$ & 0,96 & 0,95 \\
EST & 58,2 & 1,24 & 1,24 \\
\hline
\end{tabular}




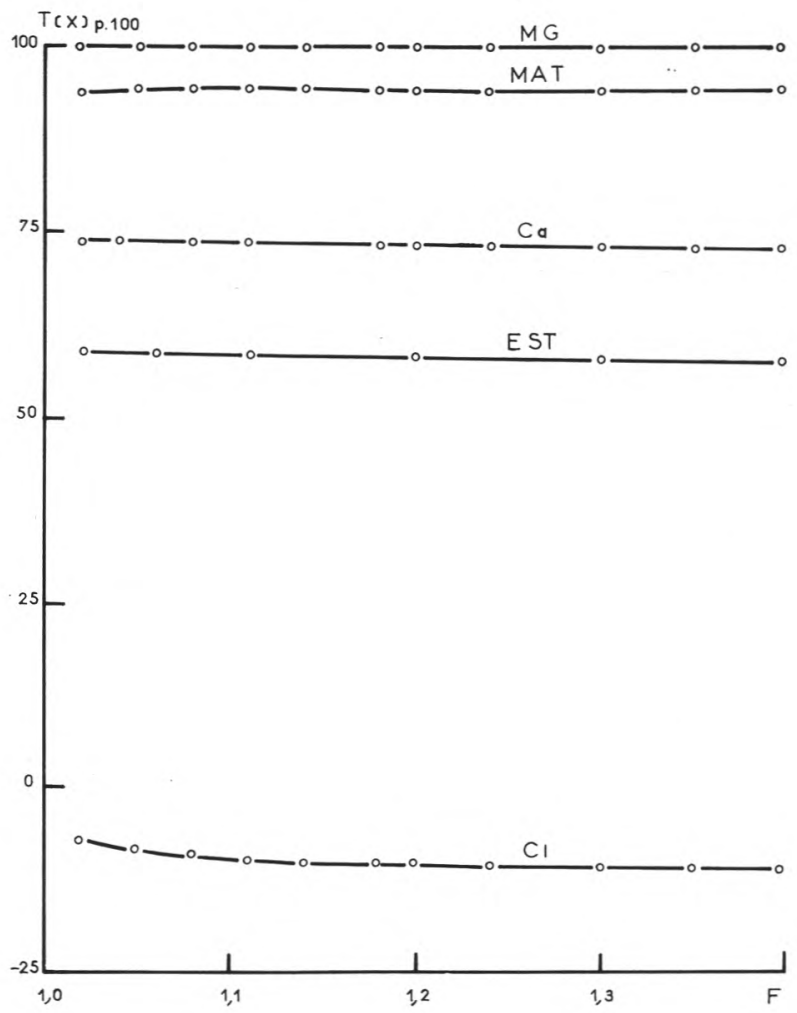

fig. 4

Taux de rétention des différents constituants du lait en fonction $\mathrm{du}$ facteur de concentration

Different milk compounds retention rates as a function of concentration factor

$\mathrm{T}(\mathrm{x})$ : taux de rétention de l'élément $\mathrm{x}$.

F : facteur de concentration volumique.
$\mathrm{T}(\mathrm{x})$ : retention rate of element $x$.

$\mathrm{F} \quad$ : volumetric concentration factor.

$\mathrm{C}_{\mathrm{r}}(\mathrm{x})=$ concentration de l'élément $\mathrm{x}$ dans le rétentat, $\mathrm{g} / \mathrm{l}$.

$\mathrm{C}_{\mathrm{l}}(\mathrm{x})=$ concentration de l'élément $\mathrm{x}$ dans le lait initial, $\mathrm{g} / \mathrm{l}$. avec :

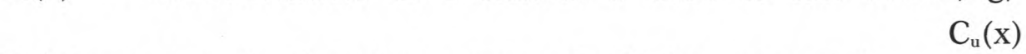

$\mathrm{T}_{\mathrm{m}}(\mathrm{x})=$ taux de rétention moyen de l'élément $\mathrm{x}=1-\frac{}{\mathrm{C}_{1}(\mathrm{x})}$

$\mathrm{C}_{\mathrm{u}}(\mathrm{x})=$ concentration de l'élément $\mathrm{x}$ dans l'ultrafiltrat, $\mathrm{g} / \mathrm{l}$.

$\mathrm{C}_{\mathrm{l}}(\mathrm{x})=$ concentration de l'élément $\mathrm{x}$ dans le lait initial, $\mathrm{g} / \mathrm{l}$. 


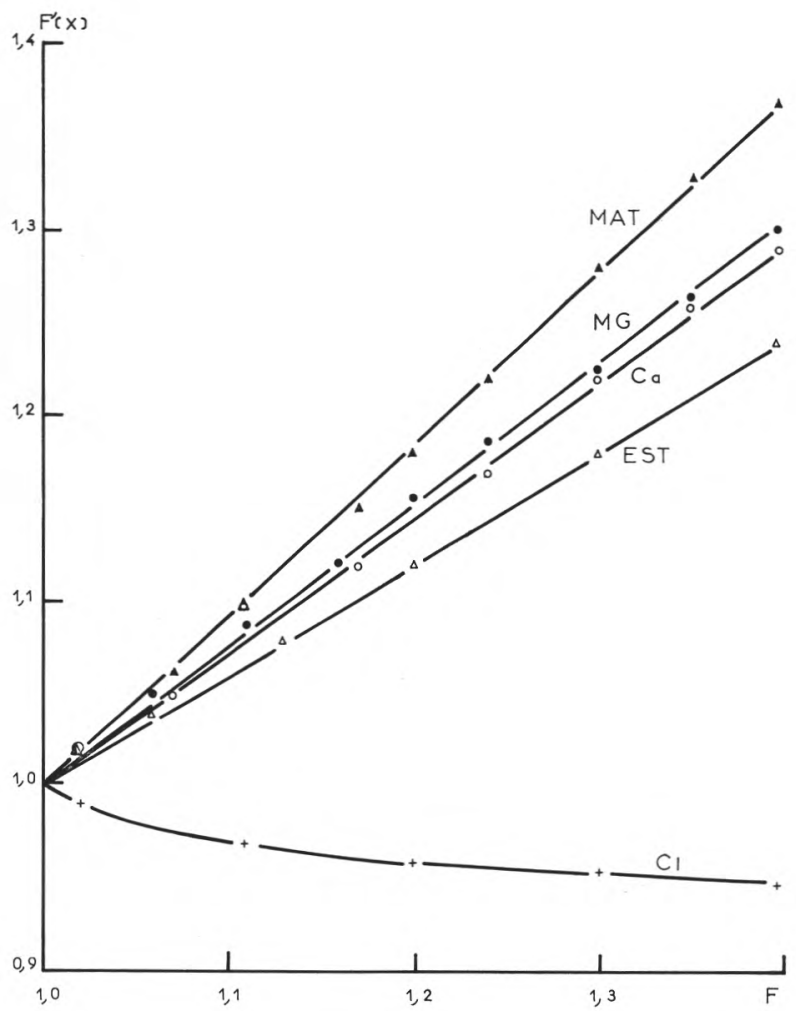

fig. 5

Facteur de concentration des différents constituants du rétentat en fonction du facteur de concentration volumique

Different retentate compounds concentration factor as a function of volumetric concentration

$F^{\prime}(x)$ : facteur de concentration de l'élément $x$.

F : facteur de concentration volumique.
$\mathrm{F}^{\prime}(\mathrm{x})$ : concentration factor of element $x$.
$\mathrm{F} \quad$ : volumetric concentration factor.

$\mathrm{F}=$ facteur de concentration volumique $=\frac{\mathrm{V}_{1}}{\mathrm{~V}_{\mathrm{r}}}$

avec :

$\mathrm{V}_{1}=$ volume de lait initial, 1 .

$\mathrm{V}_{\mathrm{r}}=$ volume de rétentat, 1 .

Connaissant le facteur de concentration volumique (F) et le taux de rétention moyen $\left(\mathrm{T}_{\mathrm{m}}\right)$ de chaque élément, nous avons calculé 
dans le tableau 3 les facteurs de concentration théoriques $F^{\prime}$ correspondants.

L'étude des taux de rétention (T) et des facteurs de concentration réels $\left(F^{\prime}\right)$, représentés respectivement sur les figures 4 et 5 en fonction de la concentration volumique $(\mathrm{F})$, et la comparaison avec les facteurs de concentration théoriques $\left(\mathrm{F}_{t}^{\prime}\right)$ dans le tableau 3 conduisent, pour chaque élément, aux remarques suivantes :

- Le taux de rétention des matières azotées confirme en valeur et en constance celui observé au laboratoire; après les premiers instants nécessaires à la formation de la couche de polarisation, pendant lesquels il s'élève légèrement, il se stabilise à une valeur correspondant au seul passage du NPN; la faible tendance à la diminution relevée ensuite s'explique par l'augmentation du gradient de concentration des MAT liée à leur accumulation au niveau de la membrane; d'ailleurs ce taux de rétention correspond théoriquement à un facteur de contentration $\left(\mathrm{F}_{t}{ }_{t}\right)$ un peu plus élevé que celui déterminé $\left(\mathrm{F}^{\prime}\right)$, ce qui tend à montrer qu'il y a fixation d'une partie des matières azotées du rétentat sur la membrane lors du colmatage, comme Lee et Merson (1975) l'ont observé par microscopie électronique pour le lactosérum.

- Il en est de même du taux de rétention en calcium qui est supérieur au pourcentage théorique de ce sel à l'état colloïdal, ce qui semble indiquer la fixation d'une fraction de sa forme soluble au niveau de la membrane et met en évidence son rôle dans le colmatage, comme l'ont récemment décrit Kessler et al. (1982). La valeur supérieure de son facteur de concentration théorique $\left(\mathrm{F}_{+}^{\prime}\right)$ par rapport à celui mesuré dans l'expérience $\left(\mathrm{F}^{\prime}\right)$ confirme également cette hypothèse. Sa diminution en fin d'opération résulte peut-être de l'accroissement de sa concentration dans le rétentat et au niveau de la membrane lorsqu'est atteint l'équilibre de formation du colmatage.

- Le taux de rétention des matières grasses est évidemment de $100 \%$ compte tenu de la méthode utilisée (cf § Matériels et méthodes) qui ne permet de doser que la matière grasse sous forme de globules gras, lesquels, étant donné leur taille, sont arrêtés par la membrane. Cependant le facteur de concentration correspondant $\left(F^{\prime}\right)$ bien inférieur à sa valeur théorique $\left(F_{t}^{\prime}\right)$ signifie qu'une partie importante de celles-ci semble disparaître du rétentat. Nous avons expliqué ce phénomène par l'éclatement d'une fraction des globules gras sous l'effet des chocs répétés, de la grande vitesse de circulation du lait et des variations importantes de pression liées à l'installation qui soustrait une partie de la matière grasse de l'analyse. L'accroissement de l'indice de lipolyse, de $0,94 \mathrm{mEq} / \mathrm{l}$ dans le lait de départ à $1,32 \mathrm{mEq} / 1$ dans le rétentat, anormal pour des substances de faibles poids moléculaires et donc dialysables (acides gras libres), vérifie en partie cette hypothèse. 
- Le taux de rétention négatif des chlorures qui correspond en fait à un taux de déperdition signifie qu'il y a un passage préférentiel de cet élément à travers la membrane. Ce comportement, qui peut paraître à première vue curieux, résulte peut-être de l'existence de charges à la surface de la membrane qui se comporterait alors comme un échangeur d'ions.

- Le faible taux de rétention en extrait sec total est la résultante de l'arrêt des grosses molécules et du passage des petites qui sont la base même du principe de l'ultrafiltration. Le facteur de concentration calculé $\left(\mathrm{F}_{t}^{\prime}\right)$ à partir de sa valeur correspond d'ailleurs à celui déterminé expérimentalement $\left(F^{\prime}\right)$. Son évolution est liée à la formation progressive de la couche de polarisation et du colmatage qui entraînent au début une diminution du passage des grosses molécules (Matières azotées solubles, Ca colloïdal) et à la fin une augmentation de la concentration locale des différents constituants qui provoque le passage en plus grande quantité des éléments solubles de petites tailles ( $\mathrm{Ca}$ soluble, $\mathrm{Cl}$ ).

Cette évolution explique également les valeurs et les variations des points cryoscopiques de la figure 6 avec la concentration du lait : dans le rétentat le point cryoscopique diminue régulièrement avec la concentration et si la pente n'est pas très importante, c'est que ce sont surtout les grosses molécules qui ont peu d'influence sur lui qui sont retenues; dans l'ultrafiltrat, le point cryoscopique de départ est évidemment plus haut étant donné sa plus grande dilution, mais il diminue ensuite de façon constante à cause du passage de petites molécules qui bien qu'en faible quantité sont les principales responsables de ses variations.

La constance du $\mathrm{pH}$ avec la concentration du lait mise en évidence dans l'ensemble malgré les contrôles bactériologiques montrant parfois une prolifération assez importante de la flore totale et (ou) des coliformes (tab. 1) indique qu'il y a un développement préférentiel de la flore hétérofermentaire. L'élévation de température en cours d'opération semble être la principale responsable de ce développement bactérien qui devrait pouvoir être évité par la suite lorsque ces paramètres seront mieux maîtrisés comme le montrent les résultats du dernier essai réalisé dans de meilleures conditions (tab. 1).

En revanche, la stérilité de l'ultrafiltrat semble être bien établie et prouve l'efficacité de la membrane qui empêche tout passage de micro-organismes, ce qui est un avantage certain pour d'éventuels traitements ultérieurs de ce produit.

En ce qui concerne le préemprésurage, nos précédents travaux (Bennasar, 1981) ont montré que sa durée et la présence de calcium provoquent de fortes variations du débit d'ultrafiltrat et nous avons mis ces effets sur le compte de l'avancement des réactions primaire enzymatique et secondaire de floculation. Dans le cas présent, nous avons testé avec la température de préemprésurage, un autre para- 


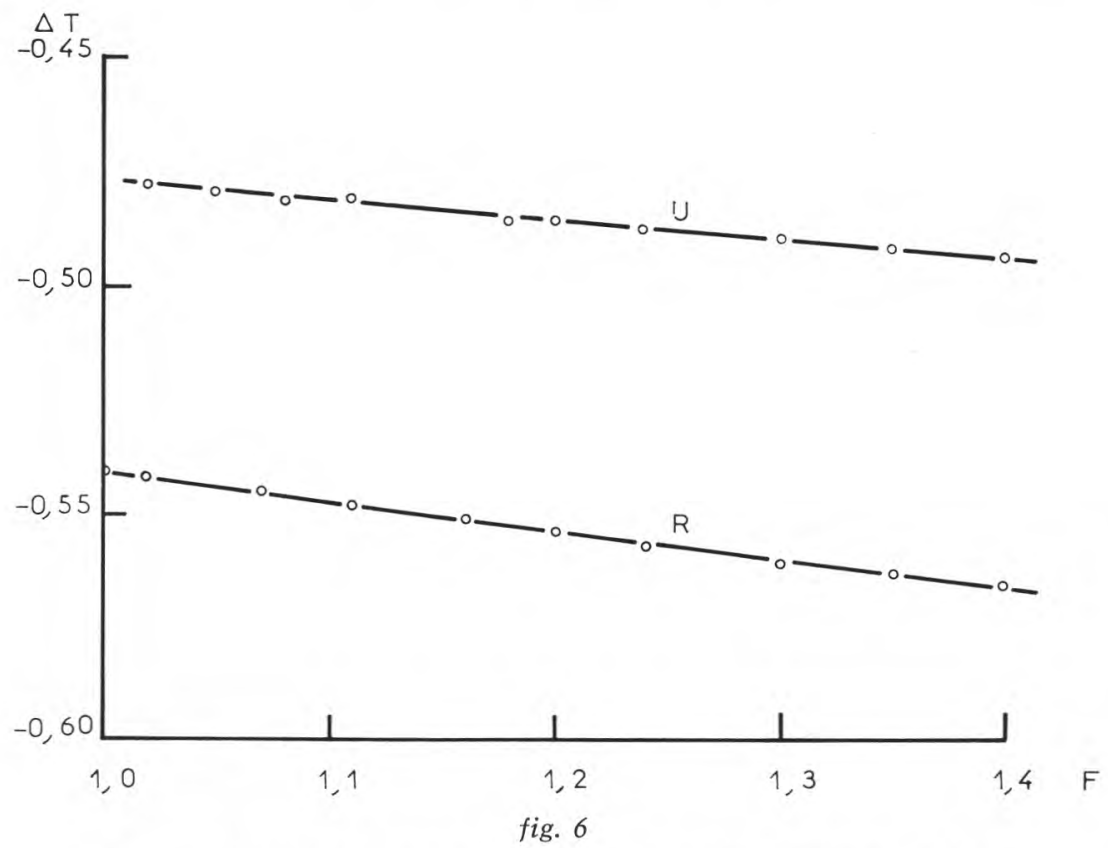

Points cryoscopiques du rétentat et de l'ultrafiltrat en fonction $\mathrm{du}$ facteur de concentration

Retentate and ultrafiltrate freezing point as a function of concentration factor

$\mathrm{U}$ : ultrafiltrat.

$\mathrm{R}$ : rétentat.

$\mathrm{F}$ : facteur de concentration volumique.

$\Delta \mathrm{T}$ : point cryoscopique.
$\mathrm{U}:$ ultrafiltrate.

$\mathrm{R}:$ retentate.

$\mathrm{F}$ : volumetric concentration factor.

$\Delta \mathrm{T}$ : freezing point.

mètre jouant sur ces mêmes vitesses de réactions comme nous l'avons déjà montré (Roongtanapirom et al. 1979).

Les résultats indiqués dans le tableau 2 confirment que tout facteur permettant d'accélérer la réaction primaire et (ou) la réaction secondaire de coagulation, sans provoquer la prise en masse du lait, entraîne une augmentation notable du débit. Il faut d'ailleurs noter à ce propos que les conditions expérimentales des essais effectués, peuvent être encore améliorées de ce point de vue (durée, température et taux d'emprésurage, enrichissement en calcium), ce qui laisse l'espoir d'atteindre des débits encore plus élevés.

Les valeurs des taux de rétention restent tout à fait correctes par rapport au témoin malgré une diminution qui va de pair avec l'augmentation du débit et que nous avons expliquée dans nos tra- 


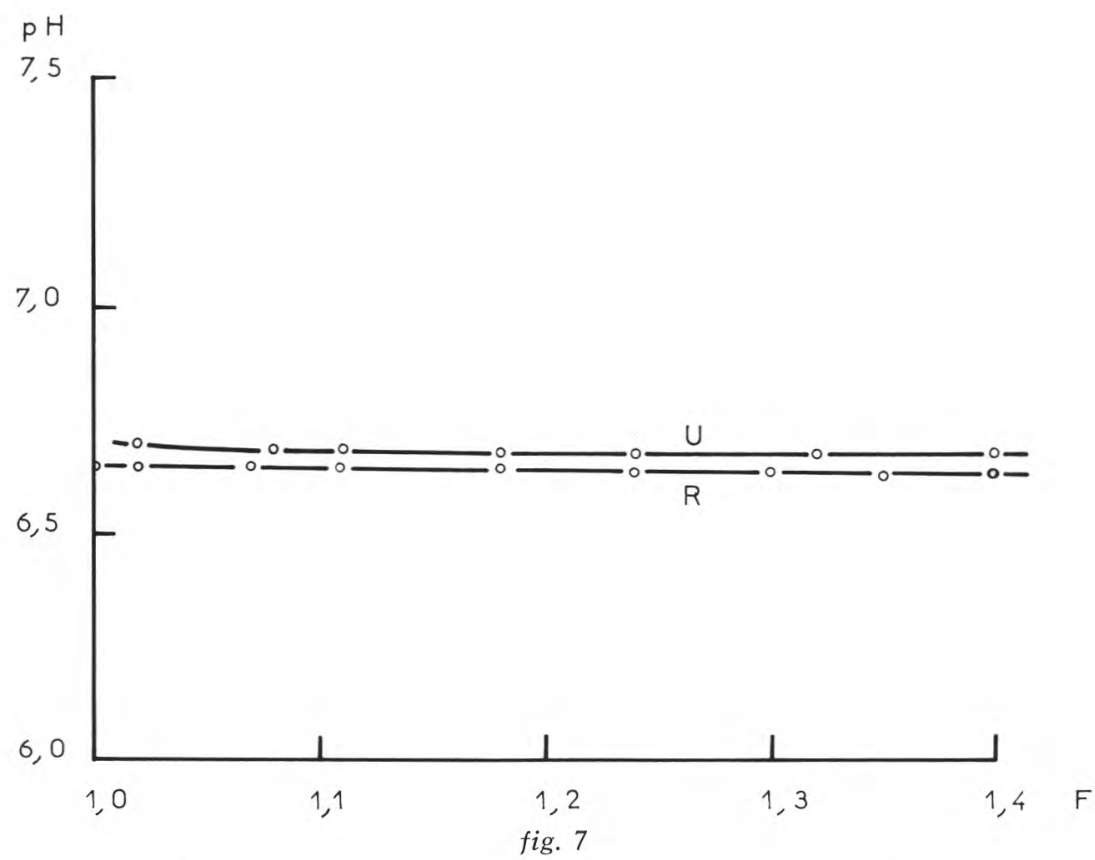

$\mathrm{pH}$ du rétentat et de l'ulltrafiltrat en fonction du facteur de concentration

Retentate and ultrafiltrate $\mathrm{pH}$ as a function of concentration factor

$\mathrm{U}$ : ultrafiltrat.

$\mathrm{R}$ : rétentat.

F : facteur de concentration volumique.

\author{
$\mathrm{U}:$ ultrafiltrate. \\ $\mathrm{R}$ : retentate. \\ $\mathrm{F}$ : volumetric concentration \\ factor.
}

vaux antérieurs (Bennasar, 1981) en faisant appel à l'augmentation de taille des espaces intermicellaires dans la couche de colmatage.

Pour ce qui est du nettoyage et de la désinfection de l'installation, les bons résultats obtenus des points de vue tenue des membranes et efficacité du traitement confirment nos précédentes observations en laboratoire et la possibilité d'appliquer à ce nouveau matériau les conditions habituelles de nettoyage dans l'industrie laitière avec toutes les garanties de sécurité et d'économie que cela représente.

\section{v. CONCLUSION}

L'ultrafiltration de lait cru entier à basse température sur membranes minérales avec un module de capacité semi-industrielle donne 
des résultats dans l'ensemble satisfaisants et attendus, compte tenu des essais réalisés précédemment dans notre laboratoire, mais elle apporte également des enseignements nouveaux. C'est ainsi que nous avons vérifié l'influence favorable de la vitesse de circulation et du préemprésurage sur les performances de l'appareil, la possibilité d'augmenter la durée de traitement et le facteur de concentration sans nuire à la qualité du produit ou aux rendements de l'opération. L'étude des taux de rétention et des facteurs de concentration des principaux constituants du lait semble montrer le rôle prépondérant du calcium et des matières azotées dans le colmatage de la couche filtrante. Enfin, la bonne tenue des membranes au nettoyage et à la désinfection est confirmée.

Quelques imperfections sont apparues au niveau de l'installation (régulation thermique défaillante, pertes de charges élevées) pouvant provoquer des accidents dans le rétentat (altération de la matière grasse, prolifération des germes); mais dans l'avenir ces inconvénients devraient pouvoir être facilement évités en modifiant la conception de l'installation et du module d'ultrafiltration et en adaptant le procédé de fabrication.

En outre, et malgré ces défauts, les fabrications fromagères réalisées avec ces rétentats s'avèrent d'ores et déjà suffisamment correctes pour nous encourager à persévérer dans cette voie et entreprendre, dès maintenant, en laboratoire l'étude de ce nouveau module d'ultrafiltration amélioré.

Nous pouvons donc conclure à la vue de l'ensemble de ces résultats que l'ultrafiltration à froid de lait entier cru, emprésuré ou non, sur membranes minérales est réalisable industriellement et susceptible d'améliorations lui permettant de s'intégrer dans les procédés classiques de fabrications de l'industrie laitière.

\section{Bibliographie}

Bennasar (M.) (1981). - Ultrafiltration du lait sur membranes minérales : amélioration des performances. Journée « Membranes artificielles et matériaux biologiques », C.N.R.S., Montpellier.

Bennasar (M.), Rouleau (D.), Mayer (R.) and Tarodo de la Fuente (B.) (1982). Ultrafiltration of milk on mineral membranes: improved performance. J. Soc. Dairy Technol., 35 (2), 43-49.

Blatt (F. W.), Dravid (A.), Michaels (A. S.) and Nelson (L.), 1970). - Solute polarization and cake formation in membrane ultrafiltration : causes, consequences and control techniques. In "Membrane Science and Technology». Ed. by Flinn (J.), Plenum Press, New York, London.

Caminade (P.), Barnier (H.) et Veyre (R.) (1982). - Réalités industrielles et perspectives de développement des membranes d'ultrafiltration de nature minérale "Carbosep ". Les Journées européennes de la filtration et de la séparation. Société Française de Filtration, Paris. 
Clifton (M.) (1980). - Polarisation de concentration dans les procédés de séparation par membrane. Journée "Membranes et leurs applications ", Université Paul-Sabatier, Toulouse.

DEJMEK (P.) (1975). - Concentration polarization in ultrafiltration of macromolécules. Thesis, Institute of Technology, Lund (Suède).

GERNEDEL (C.) (1980). - Uber die ultrafiltration von milch und die den widerstand der ablager ungsschicht beeinflussenden faktoren. Diss., TU Munchen (R.F.A.).

GLover (F. A.) and BRooKer (B. E.) (1974). - The structure of the deposit formed in the membrane during the concentration of milk by reverse osmosis. J. Dairy Res., 41 (1), 89-93.

Hiddink (J.), DE BoER (R.) and Nooy (P.F. C.) (1980), - Reverse Osmosis of dairy liquids. J. Dairy Sci., 63 (2), 204-214.

Kessler (H. G.), Gernedel (C.) and NaKanishi (K.) (1982). - The effect of low molecular weight milk constituents on the flux in ultrafiltration. Milchwissenschaft, 37 (10), 584-587.

Maubois (J. L.) et Mocovot (G.) (1971). - Préparation de fromage à partir de " pré-fromage liquide " obtenu par ultrafiltration du lait. Le Lait, 51 (508), 495-533.

Mouillet (L.), Luquet (F. M.), Nicod (H.), Boudier (J. F.) et Mathieu (H.) (1981). - La lipolyse des laits. Etude d'une méthode rapide de mesure. Le Lait, 61 (603-604), 171-186.

N'GuYen (Q. T.) (1980). - Aspects physico-chimiques des phénomènes de polarisation de concentration et de colmatage des membranes d'ultrafiltration.

III Is Journées européennes de filtration et des techniques séparatives. Société Française de Filtration, Paris.

PORTER (M. C.) (1972). - Concentration polarization with membrane ultrafiltration. Ind. Eng. Chem. Prod. Res. Develop., 11 (3), 234-248.

Porter (M. C.) and Michaels (A S.) (1971). - Membrane ultrafiltration. Chem. Tech., 1, 56-63.

Raymond (D.) et Musso (F.) (1977). - Le colmatage des membranes. Manifestations. Moyens de lutte. Dép. "Applications de l'électricité ", E.D.F., Ecuelles.

Roger (L.) et Maubois (J. L.) (1981). - Actualités dans le domaine des technologies à membrane pour la séparation et la purification des protéines laitières. Rev. Laitière Française, 400, 67-75.

Roongtanapirom (S.), Bennasar (M.) et Tarodo de la Fuente (B.) (1979). - Aptitude à la coagulation de laits pré-emprésurés secs. Le Lait, 59 (589-590), 571-588.

YAN (S. H.), HiLl (C. G.) and AmUndson (C. H.) (1979). - Ultrafiltration of whole milk. J. Dairy Sci., 62 (1), 23-40. 